Published in final edited form as:

Sex Transm Dis. 2018 April ; 45(4): e14-e17. doi:10.1097/OLQ.0000000000000774.

\title{
Higher levels of a cytotoxic protein, vaginolysin, in Lactobacillus-deficient community state types at the vaginal mucosa
}

\author{
Rebecca G. Nowak, $\mathrm{PhD}^{1,2}$, Tara M. Randis, $\mathrm{MD}^{3,4}$, Purnahamsi Desai, $\mathrm{MD}^{3}$, Xin He, $\mathrm{PhD}^{5}$, \\ Courtney K. Robinson, $\mathrm{MS}^{6}$, Jessica Rath, PhD, MPH, CHES ${ }^{7}$, Elbert D. Glover, $\mathrm{PhD}^{8}$, Adam \\ J. Ratner, $\mathbf{M D}^{3,4}$, Jacques Ravel, $\mathbf{P h D}^{6,9}$, and Rebecca M. Brotman, PhD ${ }^{2,6}$ \\ ${ }^{1}$ Institute of Human Virology, University of Maryland School of Medicine, Baltimore, MD, USA \\ ${ }^{2}$ Department of Epidemiology and Public Health, University of Maryland School of Medicine, \\ Baltimore, MD, USA \\ ${ }^{3}$ Department of Pediatrics, New York University School of Medicine, New York, NY, USA \\ ${ }^{4}$ Department of Microbiology, New York University School of Medicine, New York, NY, USA \\ ${ }^{5}$ Department of Epidemiology and Biostatistics, University of Maryland School of Public Health, \\ College Park, MD, USA \\ ${ }^{6}$ Institute for Genome Sciences, University of Maryland School of Medicine, Baltimore, MD, USA \\ ${ }^{7}$ Truth Initiative, Washington, DC, USA \\ ${ }^{8}$ Department of Behavioral and Community Health, University of Maryland School of Public \\ Health, College Park, MD, USA \\ ${ }^{9}$ Department of Microbiology and Immunology, University of Maryland School of Medicine, \\ Baltimore, MD
}

\begin{abstract}
Vaginolysin (VLY), a cytotoxic protein produced by Gardnerella vaginalis, may contribute to bacterial vaginosis (BV). Women with $G$. vaginalis, low levels of lactobacilli, history of vaginal douching, higher Nugent scores, and higher vaginal $\mathrm{pH}$ had increased VLY. Inflammatory markers were not highly expressed with increasing VLY. VLY's role in BV warrants further evaluation.
\end{abstract}

\section{Keywords}

Gardnerella vaginalis; vaginolysin; bacterial vaginosis; vaginal microbiota; 16S rRNA gene amplicon sequencing

Corresponding author: Rebecca M. Brotman, PhD, MPH, BioPark Building II, 801 West Baltimore Street, Room 627, Baltimore, MD 21201, Tel: 410-706-6767, Fax: 410-706-1482, rbrotman@ som.umaryland.edu.

Conflicts of Interest

None of the authors have conflicts of interest to declare.

Data availability: The questionnaire, VLY and immunologic marker data are available at the National Center for Biotechnology Information (NCBI) Database of Genotypes and Phenotypes (dbGaP) under Accession number phs001386.v1.p1. Metagenomic sequence data were submitted to the public NCBI Sequence Read Archive (SRA) with the accession number PRJNA391039. 
Bacterial vaginosis (BV) is a common condition associated with increased risk of sexually transmitted infections, as well as development of adverse outcomes in the female reproductive tract.(1) Although the etiology of BV remains unclear, it is characterized by an overabundance of strict or facultative anaerobic bacteria such as Gardnerella vaginalis and a decrease in Lactobacillus spp. The latter is known to produce lactic acid which drive an acidic vaginal microenvironment. $(2,3)$ Laboratory studies have demonstrated that certain strains of $G$. vaginalis can displace some Lactobacillus spp. at the mucosa (4) and BVrelated isolates of $G$. vaginalis are associated with a 2-fold increase in its cytotoxic protein, vaginolysin (VLY), compared to non-BV isolates of $G$. vaginalis in an in vitro biofilm model.(5) VLY acts by creating pores that alter cell integrity and shape and is thought to disrupt local innate immunity and thus may promote persistence of BV. $(6,7)$ It is hypothesized that BV may not induce clinical signs of inflammation because BV associated virulence factors could alter the expression of IL- $1 \beta$ and IL- 8 to prevent neutrophil recruitment. $(8,9)$ To date, expression of VLY has been limited to in vitro and animal studies.(5-7) The objective of this study was to determine the association between VLY, vaginal microbiota composition and correlates of local immunity in vivo.

Forty reproductive-aged women were enrolled as part of a previously described study at the University of Maryland School of Public Health.(10) Briefly, non-pregnant women aged 18 to 45 years who did not report using antibiotic or antimycotic drugs in the prior 30 days were recruited and completed detailed demographic and behavioral surveys. Participants self-collected a vaginal sample by inserting a double headed rayon swab (Starplex Scientific Corp., Cleveland, TN) 1-2 inches and rotated along the vaginal wall for several full circles for at least 20 seconds. Swabs were immediately snap frozen at $-20^{\circ} \mathrm{C}$ and subsequently transferred to $-80^{\circ} \mathrm{C}$ until processing. Participants determined their vaginal $\mathrm{pH}$ using a CarePlan ${ }^{\circledR} \mathrm{VpH}$ test glove. The study originally characterized the composition of the vaginal microbiota using 454 pyrosequencing of the V1-V3 hypervariable regions of the 16S rRNA genes. Vaginal microbiota were clustered into community state types (CSTs) as previously reported.(10) Thirty-seven types of human papillomavirus (HPV) were detected using the Linear Array HPV Genotyping test (Roche Molecular Diagnostics, Indianapolis, IN), of which 13 were considered high risk: $16,18,31,33,35,39,45,51,52,56,58,59$, and 68. The University of Maryland Baltimore and College Park Institutional Review Boards approved this study.

One head of the double-headed swabs was cut using sterile scissors and vortexed in $1 \mathrm{ml}$ of PBS. Aliquots $(200 \mu \mathrm{l})$ were sent to the Ratner Lab for VLY quantification and the University of Maryland Baltimore Cytokine core for the cytokine/chemokine quantification. VLY concentration was determined by ELISA as previously described (11) with the exception that a mouse monoclonal anti-VLY antibody (GenScript; 1:250 dilution) was used as the primary antibody. Total absolute bacterial abundance was estimated using a pan-bacterial 16S rRNA gene TaqMan quantitative PCR (qPCR) assay.(12) Total number of copies of 16S rRNA genes from $1 \mu \mathrm{l}$ of sample in a total reaction volume of $20 \mu \mathrm{l}$ were normalized to a standard curve (ranging from $10^{8}$ to $10^{1}$ copies/ $\mu$ in 10-fold serial dilutions) of cloned $E$. 
coli $16 \mathrm{~S}$ rRNA gene. Absolute abundance of $G$. vaginalis was calculated by multiplying the relative abundance of $G$. vaginalis obtained by $16 \mathrm{~S}$ rRNA gene sequencing data by the total bacterial 16S rRNA gene copy number generated by qPCR. A panel of 21 cytokines and chemokines were quantified from 25 $\mu$ of sample using Milliplex Multiplex Magnetic assays (Merck KGaA, Darmstadt, Germany) and the Luminex 100 system (Luminex Corp, Austin, TX). Quality control samples were included during all steps. One sample was excluded because of insufficient volume for VLY quantification. The final analytic sample size was 39.

The primary outcome variable, VLY concentration ( $\mathrm{ng} / \mathrm{ml})$, was transformed to meet the normality assumption by taking the cube root. The main independent variable was one of three CST categories previously assigned.(10) Two CSTs were dominated by Lactobacillus spp. (L. iners (CST-III) or L. crispatus (CST-I), and one lacked Lactobacillus spp. and was dominated by BV-associated bacteria including $G$. vaginalis and Atopobium vaginae (CSTIV). Other independent variables were any HPV, any high-risk HPV (HR-HPV), $\log _{10^{-}}$ transformed absolute abundance of $G$. vaginalis, Nugent's Gram's stain score (0-3, 4-6, and $7-10)$ (13), vaginal $\mathrm{pH}$ ( $\leq 4.0,4.1-4.5,4.6-5.0$, and $\geq 5.1)$, douching history, and immune markers (IL-1 $\beta$, TNF- $\alpha$, TGF- $\beta$, and IL-8). Because all transformations of the immune markers did not meet the normality assumption, IL- $1 \beta$ and TNF- $\alpha$ were dichotomized at the lower limit of detection ( $3.2 \mathrm{pg} / \mathrm{nl})$, and TGF- $\beta$ and IL- 8 were dichotomized above and below the median. Thirty-four samples were below the level of detection for IL-1 $\beta$ and/or TNF- $a$. The baseline demographics of the participants by CST were compared using Fisher's exact tests for categorical variables and analysis of variance (ANOVA) for continuous variables. Univariate linear regression models were used to estimate regression coefficients and $95 \%$ confidence intervals (CI) for the association between independent variables and VLY. Because some of the independent predictors were highly correlated with one another, 10 separate models were built (Table 1). Race, age, lifetime number of sex partners, and smoking status were evaluated as potential confounders, but none were significantly associated with CSTs and VLY. The univariate models were the most parsimonious and are presented in the table. Analyses were performed using Stata Statistical Software: Release 13 (College Station, TX: StataCorp LP).

Among the thirteen women assigned to the low-Lactobacillus CST-IV, the median age was 40 years (interquartile range 29-43). Nine (69.2\%) were African-American, ten (76.9\%) were smokers, and six (54.6\%) had ten or more lifetime sex partners. The highest concentrations of VLY were among women in CST-IV and the lowest in CST-I, a $L$. crispatus-dominated state $(\mathrm{p}<0.001)$ (Figure 1). The L. iners-dominated CST-III had an intermediate concentration of VLY which may suggest that $L$. iners can co-exist with $G$. vaginalis and its cytotoxin, and in turn, why some strains of $L$. iners have been associated with frequency and duration of BV in prior studies.(14-18)

In the univariate analyses, Lactobacillus-deficient CST-IV (p<0.01), G. vaginalis absolute abundance $(\mathrm{p}=0.01)$, increasing vaginal $\mathrm{pH}\left(\mathrm{p}_{\text {trend }}<0.01\right)$, increasing Nugent score $\left(\mathrm{p}_{\text {trend }}<0.01\right)$ and douching history $(\mathrm{p}<0.01)$ were all associated with increasing VLY concentrations. Collectively, these data suggest that $G$. vaginalis cytolysin may represent a local marker of BV. Pro-inflammatory cytokines (IL-1 $\beta$, TNF- $a$, and IL-8), as well as a 
regulator of inflammation (TGF- $\beta$ ), were not highly expressed with increasing VLY concentrations and none reached statistical significance $(p>0.05)$.

As VLY causes breakdown of tissue, we had hypothesized that VLY might allow HPV to flourish as the mucosal surface is repaired from the basal epithelial cell layers. Detection of any genotype of HPV ( $\mathrm{p}=0.50$, data not shown) and any HR-HPV were not associated with VLY concentration $(\mathrm{p}=0.73)$ (Table 1$)$.

Observations of the local immune response during BV have been inconsistent across studies. Some publications report a pro-inflammatory response with high levels of IL-1 $\beta$, TNF- $\alpha$, and IL-8 (19-22). Other studies suggest a dampened immune response that lack IL-8 and neutrophil infiltration. $(8,9)$ In a 2002 study which defined BV by Amsel's clinical criteria, Cauci et al. found the pro-inflammatory immune marker IL- 8 was positively correlated with leukocyte counts and an IgA response to a hemolysin produced by $G$. vaginalis.(23) It may be that women who have higher VLY concentration could have insufficient IL-8 concentrations to mount a humoral immune response. Certainly other bacterial species and bacterial toxins also alter local immunity but the heterogeneity in immune responses to BV merits further evaluation.

In this study, the small sample size may have limited our power to detect significant associations between the cytokines and VLY concentration. However, many of our hypothesized associations between markers of BV and VLY were detected. Second, proteins in the samples may have adhered to the swabs and could not be completely eluted during the extraction process, resulting in low or undetectable measurements of immune markers. Third, detection of VLY may vary depending on the presence of genomic variants of $G$. vaginalis and whether $G$. vaginilis is planktonic or nested in a biofilm.(24) These data were not available in the current dataset and should be considered in future studies to further elucidate the relationship between $G$. vaginalis, VLY and BV. Lastly, this cross-sectional study could not assess temporality. Despite these limitations, this study provides information on the associations between vaginal microbiota, $G$. vaginalis absolute abundance, and its cytolysin. Further studies of VLY and BV in a larger study of women are ongoing.(25)

In summary, this study adds to available in vitro findings by reporting that higher concentrations of VLY in vivo were associated with women who had a vaginal microbiota lacking lactobacilli, as well as other clinical indicators of BV. We also found that a community state type dominated by $L$. iners was often associated with VLY-producing $G$. vaginalis. Longitudinal studies are needed to assess the role of VLY in the development and persistence of $\mathrm{BV}$.

\section{Acknowledgments}

\section{Source of Funding}

All Sources of Support: This project was funded by the National Institute of Allergy and Infectious Diseases (NIAID) grants R01-AI116799 (Brotman) and R01-AI092743 (Ratner), the University of Maryland Cancer Epidemiology Alliance Joint Research Pilot Grant sponsored by the University of Maryland Greenebaum Cancer Center (Brotman), and the University of Maryland College Park / University of Maryland Baltimore Seed Grant (Brotman and Glover). 


\section{References}

1. Martin DH. The microbiota of the vagina and its influence on women's health and disease. Am J Med Sci. 2012; 343:2-9. [PubMed: 22143133]

2. Boskey ER, Cone RA, Whaley KJ, Moench TR. Origins of vaginal acidity: high D/L lactate ratio is consistent with bacteria being the primary source. Human reproduction (Oxford, England). 2001; 16(9):1809-13.

3. O'Hanlon DE, Moench TR, Cone RA. Vaginal pH and Microbicidal Lactic Acid When Lactobacilli Dominate the Microbiota. PloS one. 2013; 8(11):e80074. [PubMed: 24223212]

4. Castro J. Reciprocal Interference between Lactobacillus spp. and Gardnerella vaginalis on Initial Adherence to Epithelial Cells. International Journal of Medical Sciences. 10(9):1193-8.

5. Castro J, Alves P, Sousa C, et al. Using an in-vitro biofilm model to assess the virulence potential of Bacterial Vaginosis or non-Bacterial Vaginosis Gardnerella vaginalis isolates. Scientific Reports. 2015; 5:11640. [PubMed: 26113465]

6. Gelber SE, Aguilar JL, Lewis KL, Ratner AJ. Functional and phylogenetic characterization of Vaginolysin, the human-specific cytolysin from Gardnerella vaginalis. Journal of bacteriology. 2008; 190(11):3896-903. [PubMed: 18390664]

7. Randis TM, Zaklama J, Larocca TJ, et al. Vaginolysin drives epithelial ultrastructural responses to Gardnerella vaginalis. Infection and Immunity. 2013; 81(12):4544-50. [PubMed: 24082080]

8. Cauci S. Vaginal Immunity in Bacterial Vaginosis. Curr Infect Dis Rep. 2004; 6(6):450-6. [PubMed: 15538982]

9. Cauci S, Culhane JF, Di Santolo M, McCollum K. Among pregnant women with bacterial vaginosis, the hydrolytic enzymes sialidase and prolidase are positively associated with interleukin-1beta. Am J Obstet Gynecol. 2008; 198(1):132e1-7. [PubMed: 17714681]

10. Brotman RM, He X, Gajer P, et al. Association between cigarette smoking and the vaginal microbiota: a pilot study. BMC Infect Dis. 2014; 14:471. [PubMed: 25169082]

11. Randis TM, Kulkarni R, Aguilar JL, Ratner AJ. Antibody-based detection and inhibition of vaginolysin, the Gardnerella vaginalis cytolysin. PLoS ONE. 2009; 4(4):e5207. [PubMed: 19370149]

12. Liu CM, Aziz M, Kachur S, et al. BactQuant: An enhanced broad-coverage bacterial quantitative real-time PCR assay. BMC microbiology. 2012; 12 56-2180-12-56.

13. Nugent R, Krohn M, Hillier S. Reliability of diagnosing bacterial vaginosis is improved by a standardized method of Gram stain interpretation. J Clin Microbiol. 1991; 29:297-301. [PubMed: 1706728]

14. Macklaim JM, Gloor GB, Anukam KC, Cribby S, Reid G. At the crossroads of vaginal health and disease, the genome sequence of Lactobacillus iners AB-1. Proceedings of the National Academy of Sciences. 2011; 108(Suppl 1):4688-95.

15. McMillan A, Macklaim JM, Burton JP, Reid G. Adhesion of Lactobacillus iners AB-1 to Human Fibronectin: A Key Mediator for Persistence in the Vagina? Reproductive sciences (Thousand Oaks, Calif). 2013; 20(7):791-6.

16. Verstraelen H, Verhelst R, Claeys G, De BE, Temmerman M, Vaneechoutte M. Longitudinal analysis of the vaginal microflora in pregnancy suggests that L. crispatus promotes the stability of the normal vaginal microflora and that L. gasseri and/or L. iners are more conducive to the occurrence of abnormal vaginal microflora. BMC Microbiol. 2009; 9:116. [PubMed: 19490622]

17. Srinivasan S, Liu C, Mitchell CM, et al. Temporal Variability of Human Vaginal Bacteria and Relationship with Bacterial Vaginosis. PLoS ONE. 2010; 5(4):e10197. [PubMed: 20419168]

18. Witkin SS, Mendes-Soares H, Linhares IM, Jayaram A, Ledger WJ, Forney LJ. Influence of vaginal bacteria and D- and L-lactic acid isomers on vaginal extracellular matrix metalloproteinase inducer: implications for protection against upper genital tract infections. MBio. 2013; 4(4)

19. Platz-Christensen JJ, Mattsby-Baltzer I, Thomsen P, Wiqvist N. Endotoxin and interleukin-1 alpha in the cervical mucus and vaginal fluid of pregnant women with bacterial vaginosis. Am J Obs Gyn. 1993; 169(5):1161-6.

20. Sturm-Ramirez K, Gaye-Diallo A, Eisen G, Mboup S, Kanki PJ. High levels of tumor necrosis factor-alpha and interleukin-1beta in bacterial vaginosis may increase susceptibility to human 
immunodeficiency virus. The Journal of infectious diseases. 2000; 182(2):467-73. [PubMed: 10915077]

21. Hedges SR, Barrientes F, Desmond RA, Schwebke JR. Local and systemic cytokine levels in relation to changes in vaginal flora. The Journal of Infectious Diseases. 2006; 193(4):556-62. [PubMed: 16425135]

22. Zariffard MR, Novak RM, Lurain N, Sha BE, Graham P, Spear GT. Induction of tumor necrosis factor- alpha secretion and toll-like receptor 2 and 4 mRNA expression by genital mucosal fluids from women with bacterial vaginosis. J Infect Dis. 2005; 191(11):1913-21. [PubMed: 15871126]

23. Cauci S, Guaschino S, Driussi S, De Santo D, Lanzafame P, Quadrifoglio F. Correlation of local interleukin-8 with immunoglobulin A against Gardnerella vaginalis hemolysin and with prolidase and sialidase levels in women with bacterial vaginosis. The Journal of Infectious Diseases. 2002; 185(11):1614-20. [PubMed: 12023767]

24. Castro J, França Â, Bradwell KR, Serrano MG, Jefferson KK, Cerca N. Comparative transcriptomic analysis of Gardnerella vaginalis biofilms vs. planktonic cultures using RNA-seq. npj Biofilms and Microbiomes. 2017 1-0.

25. Desai, PVRT., Sapra, E., Sapra, KJ., Shutak, C., Gelber, SE., Ratner, AJ. Detection of vaginolysin as a new diagnostic tool for bacterial vaginosis. Pediatric Academic Societies' Annual Meeting; Vancouver, BC. 2014. 


\section{Short summary}

Women with indicators of bacterial vaginosis, including a vaginal microbiota lacking Lactobacillus spp., had higher in vivo concentrations of vaginolysin, a cytotoxic protein. 


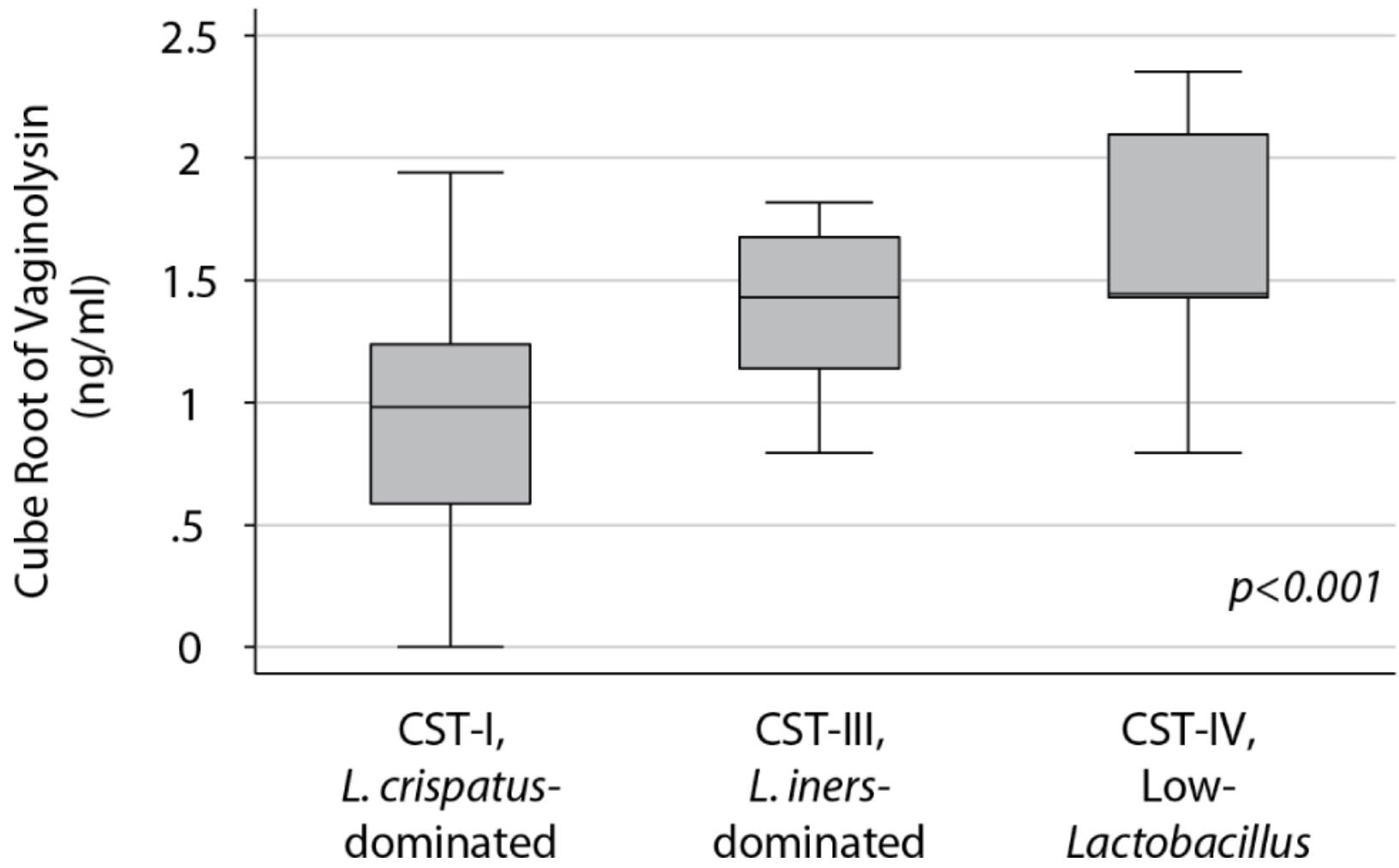

Figure 1.

Vaginolysin ( $\mathrm{ng} / \mathrm{ml})$ by community state type

Note: Analysis of variance (ANOVA) was used to test differences in vaginolysin by community state type. 


\section{Table 1}

Univariate analysis of risk factors associated with vaginolysin

\begin{tabular}{|c|c|c|c|}
\hline \multirow[b]{2}{*}{ Model } & \multirow[b]{2}{*}{ Variable } & \multicolumn{2}{|c|}{ Regression coefficient* } \\
\hline & & Unadjusted $(95 \% \text { CI })^{* *}$ & $\mathbf{P}$ \\
\hline \multirow[t]{4}{*}{1} & Vaginal community state type (CST) & & \\
\hline & CST-I, L. crispotus-dominated & Ref. & \\
\hline & CST-III, L. iners-dominated & $0.48(0.06,0.90)$ & 0.03 \\
\hline & CST-IV, Loctobocillus-deficient & $0.75(0.39,1.11)$ & $<0.01$ \\
\hline 2 & Gardnerella vaginalis ( $\log _{10}$ counts) & $0.19(0.05,0.33)$ & 0.01 \\
\hline \multirow[t]{3}{*}{3} & HR-HPV & & \\
\hline & No & Ref. & \\
\hline & Yes & $0.07(-0.33-0.47)$ & 0.73 \\
\hline \multirow[t]{4}{*}{4} & Nugent Gram stain score & & $<0.01$ \\
\hline & $0-3$ & Ref. & \\
\hline & $4-6$ & $0.43(-0.13,1.00)$ & \\
\hline & $7-10$ & $0.65(0.24,1.06)$ & \\
\hline \multirow[t]{5}{*}{5} & Vaginal $\mathrm{pH}$ & & $<0.01$ \\
\hline & $\leq 4.0$ & Ref. & \\
\hline & $4.1-4.5$ & $0.05(-0.60,0.70)$ & \\
\hline & $4.6-5.0$ & $0.45(-0.04,0.95)$ & \\
\hline & 25.1 & $0.70(0.32,1.07)$ & \\
\hline \multirow[t]{3}{*}{6} & History of douching & & \\
\hline & No & Ref. & \\
\hline & Yes & $0.53(0.15-0.91)$ & $<0.01$ \\
\hline \multirow[t]{3}{*}{7} & $\mathrm{IL}-1 \beta^{\dagger}$ & & \\
\hline & $\geq 3.20$ (LLOD) & Ref. & \\
\hline & 23.20 & $0.10(-0.28,0.48)$ & 0.61 \\
\hline \multirow[t]{3}{*}{8} & TNF- $a^{\dagger}$ & & \\
\hline & $<3.20$ (LLOD) & Ref. & \\
\hline & $>3.20$ & $-0.20(-0.67,0.27)$ & 0.39 \\
\hline \multirow[t]{3}{*}{9} & TGF- $\beta^{t+t}$ & & \\
\hline & $<47.39$ & Ref. & \\
\hline & $\geq 47.39$ & $-0.30(-0.67,0.07)$ & 0.10 \\
\hline \multirow[t]{3}{*}{10} & $\mathrm{IL}-8^{\dagger}$ & & \\
\hline & $<340.88$ & Ref. & \\
\hline & $\geq 340.88$ & $-0.19(-0.56,0.19)$ & 0.32 \\
\hline
\end{tabular}

Sex Transm Dis. Author manuscript; available in PMC 2019 April 01. 
Note: HR-HPV, high risk human papillomavirus; $a$, alpha; $\beta$, Beta; P, p-value; LLOD, lower limit of detection; CI, confidence interval; Ref., reference.

Vaginolysin concentration transformed with cube root to satisfy normality assumption

** Unadjusted values are reported because race, age, lifetime number of sex partners, and smoking status did not confound the main associations and the more parsimonious models were reported.

${ }^{+} \mathrm{pg} / \mathrm{ml}$

tatent TGF- $\beta 1$ 